\title{
Use of Infrared Spectroscopy and Near Infrared Hyperspectral Images to Evaluate Effects of Different Chemical Agents on PET Bottle Surface
}

\author{
Alianda Dantas de Oliveira ${ }^{a}$, Vitor Hugo da Silva ${ }^{b}$, Maria Fernanda Pimentel ${ }^{c}$, Glória Maria Vinhas ${ }^{a}$, \\ Celio Pasquinid, Yêda Medeiros Bastos de Almeida ${ }^{*}$ *i] \\ ${ }^{a}$ Universidade Federal de Pernambuco, Centro de Ciências Exatas e da Natureza, Programa de Pós \\ Graduação em Ciências dos Materiais, Av. Jornalista Anibal Fernandes, Cidade Universitária, 50.740- \\ 560, Recife, PE, Brasil \\ ${ }^{b}$ Universidade Federal de Pernambuco, Departamento de Química Fundamental - DQF, Av. Prof. \\ Moraes Rego, 1235, Cidade Universitária, 50670-901, Recife, PE, Brasil \\ cUniversidade Federal de Pernambuco, Departamento de Engenharia Quimica, Av. Prof. Arthur de Sá, \\ Cidade Universitária, 50740-521, Recife, PE, Brasil \\ ${ }^{d}$ Universidade Estadual de Campinas, Instituto de Química - IQ, Caixa Postal 6154, 13083-970, \\ Campinas, SP, Brasil
}

Received: October 20, 2017; Revised: June 12, 2018; Accepted: July 09, 2018

PET - poly (ethylene terephthalate) - bottle surfaces were exposed to chemical agents and evaluated with middle infrared spectroscopy (MIR) and near infrared hyperspectral imaging (HSI-NIR). Images $(928-2.524 \mathrm{~nm})$ were acquired at different times from bottle surfaces exposed to alkaline, acidic and ethanolic media. Savitzky-Golay smoothing ( $2^{\text {nd }}$ order polynomial and 5-point window) combined with Standard Normal Variate (SNV) was used for pre-processing data. Scores from principal component analysis (PCA) on images showed discrimination of PET according to exposure time, particularly when employing $\mathrm{NaOH}$. After the first two hours of chemical exposure, significant contribution of the $(-\mathrm{C}-\mathrm{O}+\mathrm{OH})$ combination band indicated formation of glycol ethylene on the PET surface, which was confirmed from PCA loading plots. MIR reflectance spectra ranged from 4.000 to $650 \mathrm{~cm}^{-1}$. Changes in spectral intensities could be associated with depolymerization of PET when exposed to $\mathrm{NaOH}$. These were confirmed with PCA and attributed to the carboxylate group of terephthalate.

Keywords: Poly (ethylene terephthalate), chemical attack, infrared spectroscopy, hyperspectral images.

\section{Introduction}

Chemical attacks in polymers happen when a chemical reaction occurs on the polymeric material by contact with chemical agents. In a chemical attack, the polymeric material may suffer modifications such as color variation and loss/ gain of mass. This kind of chemical attack is commonly confounded with Environmental Stress Cracking (ESC) because both cause cracking and brittleness of the material. However, in the ESC process, both chemical exposure and a tensile test (physical phenomenon) are included ${ }^{1,2}$.

A large number of chemical agents (aggressive fluids) when in contact with polymeric material can cause ESC, product contaminations within packages, as well as depolymerization. The literature includes reports on a huge number of chemical agents and their modifications on polymeric materials ${ }^{3-10}$. Muller et al. examined contamination in a sealed cola bottle in contact with toluene, xylene, cyclohexane, methylcyclohexane and turpentine. The authors showed that the organic solvents penetrated the bottle without any apparent cola leakage and they observed bottle cracks due to internal gasification pressure and the chemical agent action ${ }^{9}$. Gárcia et al. studied

*e-mail: yeda@ufpe.br
PET degradation using ethylene carbonate and potassium hydroxide, at $130^{\circ} \mathrm{C}$ over a period of $24 \mathrm{~h}^{10}$.

Most of the studies focus on ESC, such as that conducted by Al-Saidi et al. which investigated polycarbonate resistance in the ESC process using isopropyl alcohol, ethylene glycol monomethyl ether and methanol. The results evidenced failures in the polycarbonate during the ESC process resulting from all chemical agents tested, with ethylene glycol monomethyl ether the most aggressive chemical agent among them ${ }^{4}$. Poly (ethylene terephthalate) - PET has been extensively used in studies focused on the evaluation of despolimerization, due to its applicability as packing material for foods and because it is a very widespread material. In these works, solutions such as acetic acid, sulphur acid, methanol, sodium acetate and sodium hydroxide $(\mathrm{NaOH})$ have been the most commonly used $^{1,6,7,11}$. Among these solutions, sodium hydroxide $(\mathrm{NaOH})$ has shown to be the most aggressive PET chemical agent, degrading around $98 \%$ of the polymeric material at a controlled temperature $\left(100^{\circ} \mathrm{C}\right)$ and atmospheric pressure for $5 \mathrm{~h}$, as demonstrated by Mancini and Zanin ${ }^{6}$. Ng et al. ${ }^{12}$ evaluated chemical attack in nonwoven PET fibrous with $\mathrm{NaOH}$ solution at $70^{\circ} \mathrm{C}$ for up to $120 \mathrm{~h}$. The study demonstrated hydrolysis up to $70 \%$, increasing the roughness of the PET 
surface. The results also demonstrated that PET treatment with $\mathrm{NaOH}$ could be used to produce fibers with predictable porosity, pore size, and fiber diameter. Overall, degree and rate of hydrolysis in the nonwoven PET fibrous depended on the degrees of crystallinity. These studies have shown that the PET amorphous region, which has a higher free volume and, consequently, shows higher swelling of the chemical agent, can provide preferential paths for the depolymerization process $^{1}$. In these depolymerization studies, the PET bottle was cut and soaked in the chemical agent solutions, where side absorption could occur. Moreover, temperatures equal or greater than to $70^{\circ} \mathrm{C}$ were used to improve the process conditions $^{6,11}$.

Generally, studies of the chemical attacks in polymeric materials are evaluated by morphologic aspects using scanning electron microcopy (SEM), atomic force microscopy

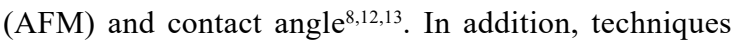
such as Differential Scanning Calorimetry (DSC) and Thermogravimetric Analysis (TGA) are commonly used to characterize polymeric materials ${ }^{14-16}$. However, these techniques are destructive and, consequently, do not make it possible to evaluate the exposure times with chemical agents using the same sample. For this purpose, rapid and non-destructive analytical methods are more attractive to explore the chemical attacks on polymeric materials over time. Vibrational spectroscopy such as near- (NIR) and middle-infrared (MIR) can satisfy these criteria. In the middle spectral range, absorption bands are found that are associated to fundamental vibration transitions. In the NIR region, the energy range is higher than necessary to promotes molecules only to their lowest excited vibrational states (fundamental vibrational transition) and the spectral occurrences are mainly due overtones and combination absorption bands related to N-H, C-H, O-H and S-H bonds. These techniques have been widely used for polymer material characterization and, when associated with chemometric tools, are powerful for qualitative and quantitative analyses ${ }^{16-20}$. Ozzetti et al. (2002) determined the tacticity of polypropylene films using the MIR spectroscopy and multivariate techniques ${ }^{17}$. Ghita et al. (2008) monitored the polymer color in-line during injection molding using NIR and multivariate calibration models ${ }^{18}$. Caetano et al. (2013) determined the tensile strength and tensile modulus of PET films using multivariate calibration models ${ }^{19}$. Qualitative and quantitative analyses of plasticizers in polylactide (PLA) using MIR and independent component analysis - ICA was made by Kassouf et al. $(2016)^{20}$.

A relatively new technique, NIR hyperspectral imaging (NIR-HSI), which provides spatial and spectral information about the sample, has advanced and its applicability in the polymers field has consequently grown. In the NIR-HSI systems, a NIR spectrum is acquired for each image pixel and a hypercube (3-way) is generated containing the spatial (x-and y-coordinates) and spectral information (z-coordinate, wavelengths). Hyperspectral images provide a large amount of information which makes the analysis a complex task. However, multivariate data analysis provides efficient tools to extract useful information from these images. Conventional chemometric techniques can be applied to the images such as principal component analysis (PCA) through the unfolding of the hypercube into a two way (2-way) matrix. With the advent of hyperspectral images as an analytical technique it became possible to obtain spatial and spectral (and thus chemical) information about a sample. Excellent reviews about HSI and its analysis can be found in the literature ${ }^{21,22}$.

A number of works have demonstrated the NIR-HSI potential in the polymers field for: crystallinity variations in HDPE (high density polyethylene), LDPE (low density polyethylene) and PP (polypropylene) ${ }^{23}$; contamination of secondary plastics ${ }^{24}$; miscibility of poly (3-hydroxybutyrate) blends - PHB and PLA ${ }^{25}$; classification of polymers (PET and PVC) in different life cycles ${ }^{26}$; maps of the content of diethyleneglycol (DEG) and terminal carboxylic groups (GCT) in a PET fiber sample; ${ }^{27}$ and visualization of hydrolysis in polylactide (PLA) ${ }^{28}$. Although this technique can potentially reveal the distributions of the chemical attack on a polymeric surface, as far as we know, NIR-HSI has not been used to identify areas where the attack has occurred nor to evaluate possible preferential paths for these chemical attacks.

Considering the aspects mentioned, the present work aims to evaluate the effect of different chemical agents on a PET bottle surface over time using rapid and non-destructive techniques: NIR-HSI and MIR spectroscopy. HSI-NIR was employed to observe if there were preferential paths on the PET surface for absorption of the chemical agents. Using NIR images associated to chemometric techniques allowed identification of areas of the polymer surface where degradation had occurred. Attenuated reflectance spectroscopy in the infrared middle range was also employed to observe surface changes, as the infrared beam only penetrates about 2 micrometers into the sample. As mentioned, neither technique has been employed to evaluate the effect of chemical agents on the polymer surfaces. This work uses a new approach where only one side of the PET surface is in contact with the chemical agents without contact with lateral areas, avoiding the effect of lateral absorptions. Moreover, to date no study has evaluated chemical attacks on PET over a long period and under mild temperature conditions, using the same polymer sample.

\section{Experimental}

\subsection{Materials}

Samples were collected from used, non-colored soft drink PET bottles. The bottles were cut in its lateral region into rectangular pieces $(75 \times 55 \mathrm{~mm})$ totaling 12 samples. The PET bottle samples were washed with neutral soap and subsequently dried with absorbent paper. 
Ethanol (analytical grade, Merck), phosphoric acid (purity $85 \%$, Chemo), sodium hydroxide pellets (purity $99 \%$, Merck), ascorbic acid (purity $99 \%$, Synth), sodium bicarbonate (purity $99.7 \%$, Synth) and citric acid (purity $99.5 \%$, Vetec) were used as the chemical agents. These chemical agents were selected because they have been reported to cause superficial changes and failures in PET materials (ethanol, sodium hydroxide and sodium bicarbonate). Some of these agents, as well, are found in commercialized

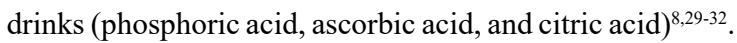
Solutions using these chemical agents were prepared in molar concentrations of $3 \mathrm{~mol}$. $\mathrm{L}^{-1}$. The experiments were performed in duplicate.

\subsection{Exposure of PET samples to the chemical agents}

The PET samples were exposed to each chemical agent solution at room temperature $\left(22 \pm 2^{\circ} \mathrm{C}\right)$ for different times: 2 h, 4 h, 8 h, 24 h, 48 h, 72 h, 96 h, 15 days, 30 days and 60 days.

The exposure test was performed using accessories (totaling 6) designed by the authors and produced by Altecval Company (Figure 1). This accessory consisted of a Teflon cylinder $(50 \mathrm{~mm})$ with an internal diameter of $41 \mathrm{~mm}$, two aluminum plates ( $80 \mathrm{~mm} \times 80 \mathrm{~mm}$ and $5 \mathrm{~mm}$ thickness) and screws. The PET samples were placed between the aluminum plates and the Teflon cylinder. All chemical agents used in this work were inserted through the top of the accessory,

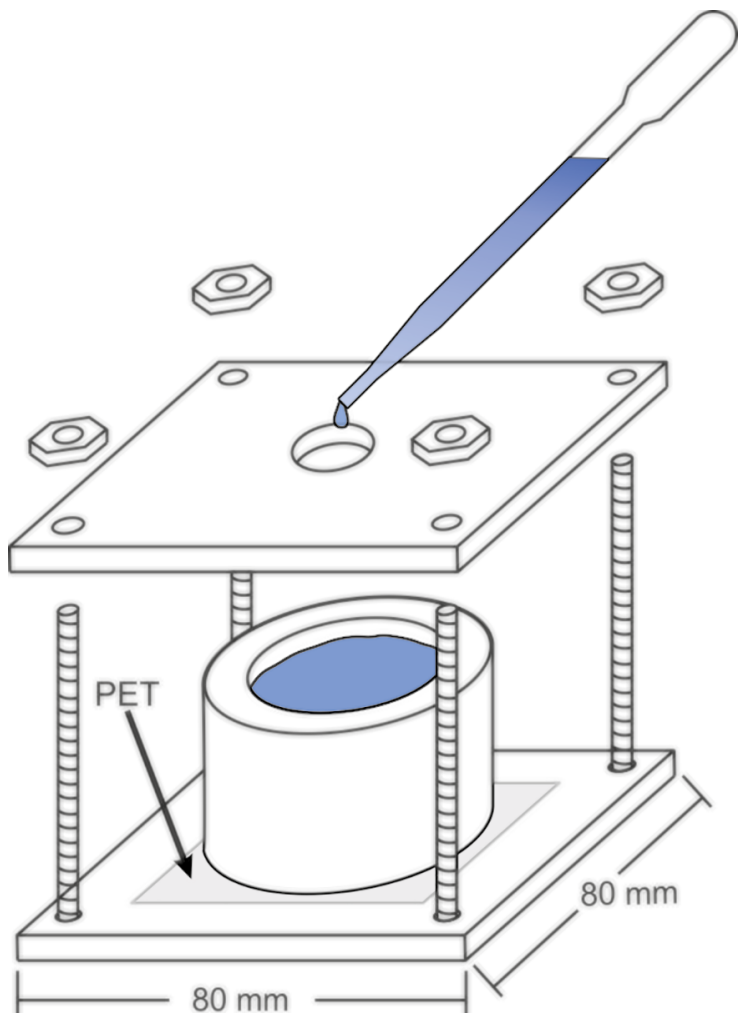

Figure 1. Accessory used for the PET chemical attack test. using a disposable Pasteur pipette as shown in Figure 1. This accessory was used to guarantee that only the surface of the film was in contact with the chemical agent.

After each exposure time, the PET sample was washed with distilled water and dried with absorbent paper. Finally, HSI-NIR images and MIR spectra were acquired for each PET samples. The PET sample after the spectral measurement was placed back in the accessory for an additional period of time, i.e., the same sample was analyzed in all exposure times. Image and MIR spectra were also acquired before the exposure test. The PET bottle samples attacked by a sodium hydroxide solution was also evaluated using the MIR spectroscopy, to enable a better investigation of the polymer surface changes.

\subsection{Spectral and Scanning Electron Microscopy (SEM) Analysis}

Near infrared hyperspectral images (HSI-NIR) were acquired using a SisuChema (Specim) hyperspectral camera. This camera measures cross-sections of the sample (line). The spectral range used was $928-2.524 \mathrm{~nm}$ (256 variables), with spectral resolution and spectral sampling/pixel of 10 $\mathrm{nm}$ and $6.3 \mathrm{~nm}$, respectively. The images were acquired with a pixel size of $150 \times 150 \mu \mathrm{m}$. This type of camera enables image acquisition of the entire sample $(75 \times 55 \mathrm{~mm})$ very quickly, around 7 seconds.

Middle Infrared spectra were acquired using the FTIR Spectrum 400 spectrometer (Perkin Elmer) with a diamond Universal Attenuated Total Reflectance (UATR) accessory. As stated by the manufacture, the penetration depth of the IR radiation is around $2 \mu \mathrm{m}$ at $1.000 \mathrm{~cm}^{-1}$. The spectra were obtained in the range of 4.000 to $650 \mathrm{~cm}^{-1}$, with a resolution of $4 \mathrm{~cm}^{-1}$ and average of 32 scans per spectrum. Eight spectral measurements were taken at different places on each PET sample for each exposure time.

The SEM images were obtained with a FEI Quanta FEG 250 microscope after gold metallization of the PET samples. Micrographs were acquired with $2.000 \mathrm{x}$ magnification and $2.00 \mathrm{Kv}$ voltage

\subsection{Data Pre-processing and Analysis}

The NIR images were acquired from the $75 \times 55 \mathrm{~mm}$ samples. Anomalous pixels (dead pixels) were also removed from the HSI-NIR. Dead pixels are common in hyperspectral images, caused by detector failures during image acquisition These anomalous pixels were replaced by the average values of neighbor pixels to improve the image quality. Following this, the noise regions of the spectra $(928$ to $1.550 \mathrm{~nm}$ and 2.519 to $2.524 \mathrm{~nm}$ ) were excluded. To compare the effects of the different chemical agents, the images were cropped ( $200 \times 200$ pixels), with the objective of selecting the region of interest for this work, corresponding to the area of the PET image that had been exposed to the chemical agent. 
Different pre-processing strategies were applied in the HSI-NIR and MIR spectra to correct the noise and scattering effects. The pre-processing techniques used in this work were Standard Normal Variate (SNV), Multiplicative Scattering Correction (MSC) and smoothing with Savitzky-Golay (SG) filtering. Furthermore, the first derivative (Savitzky-Golay, $2^{\text {nd }}$ second order polynomial using different window points) was also evaluated. ${ }^{33}$.

HSI-NIR and MIR spectra were analyzed using Principal Components Analysis (PCA), a well-known chemometric method for exploratory analysis of multivariate data. This unsupervised pattern recognition technique was used in this work to identify possible preferential paths for the attack of the chemical agents (HSI-NIR), as well as spectral changes during exposure (HSI-NIR and MIR spectroscopy). The purpose of the PCA is to evaluate the natural behavior of the samples in a multivariate dataset. This technique reduces the data's dimensions and represents the maximum variance of the dataset in a new orthogonal axes system (principal components - PC). The new axes are a linear combination of the original variables, allowing the visualization of the sample features in a smaller dimension. The samples projections in the new axes are called scores and they describe the differences and similarities among the samples. The contribution of each variable in the new orthogonal axes is called loadings and indicates how much each original variable contributes to a given $\mathrm{PC}^{33}$. When applied to images, the data set needs to be unfolded previously to a $2 \mathrm{D}$ matrix where each pixel is considered as a sample, as demonstrated in Figure 2. In that case, the scores and loadings contain the spatial (pixels) and spectral information, respectively. After PCA models, the image is refolded (3D matrix), resulting in images for each PC with its pixel distributions and its explained data variability, as well as the loadings profiles. In this work, therefore, PCA was able to distinguish pixels in the hyperspectral image that might be attacked by the agents over time using the scores plot and to visualize the spectral ranges associated with these chemicals attack (loadings).

Hierarchical Cluster Analysis (HCA) was also employed in this work to evaluate possible clustering of the samples according to the time exposition to the chemical agents. To generate the dendogram, single linkage and Euclidian distances were used.

All chemometric treatment was performed using Matlab (R2010a 7.10.0.499, MathWorks) and HYPER-tools software ${ }^{22}$.

\section{Results and Discussion}

\subsection{Visual aspect of PET samples}

Visual aspect analysis was made with the PET samples after different exposure times and chemical agents. Only the PET in contact with the $\mathrm{NaOH}$ solution showed visual modification after 30 days of exposition, as can be seen in Figure 3. A whitish color (Figure 3a) was observed in the PET samples and this characteristic was intensified after 60 days of exposition (Figure 3c). This feature observed in the PET samples treated with the $\mathrm{NaOH}$ solution may be associated with the sodium terephthalate formation from the hydrolysis process which can occur on a PET surface, as confirmed by chemometric analysis.

\subsection{NIR Spectra of PET images}

Figure 4 shows the raw mean spectrum of the PET images before exposure to the chemical agents. As mentioned, the noise regions of the spectra were removed and a spectral

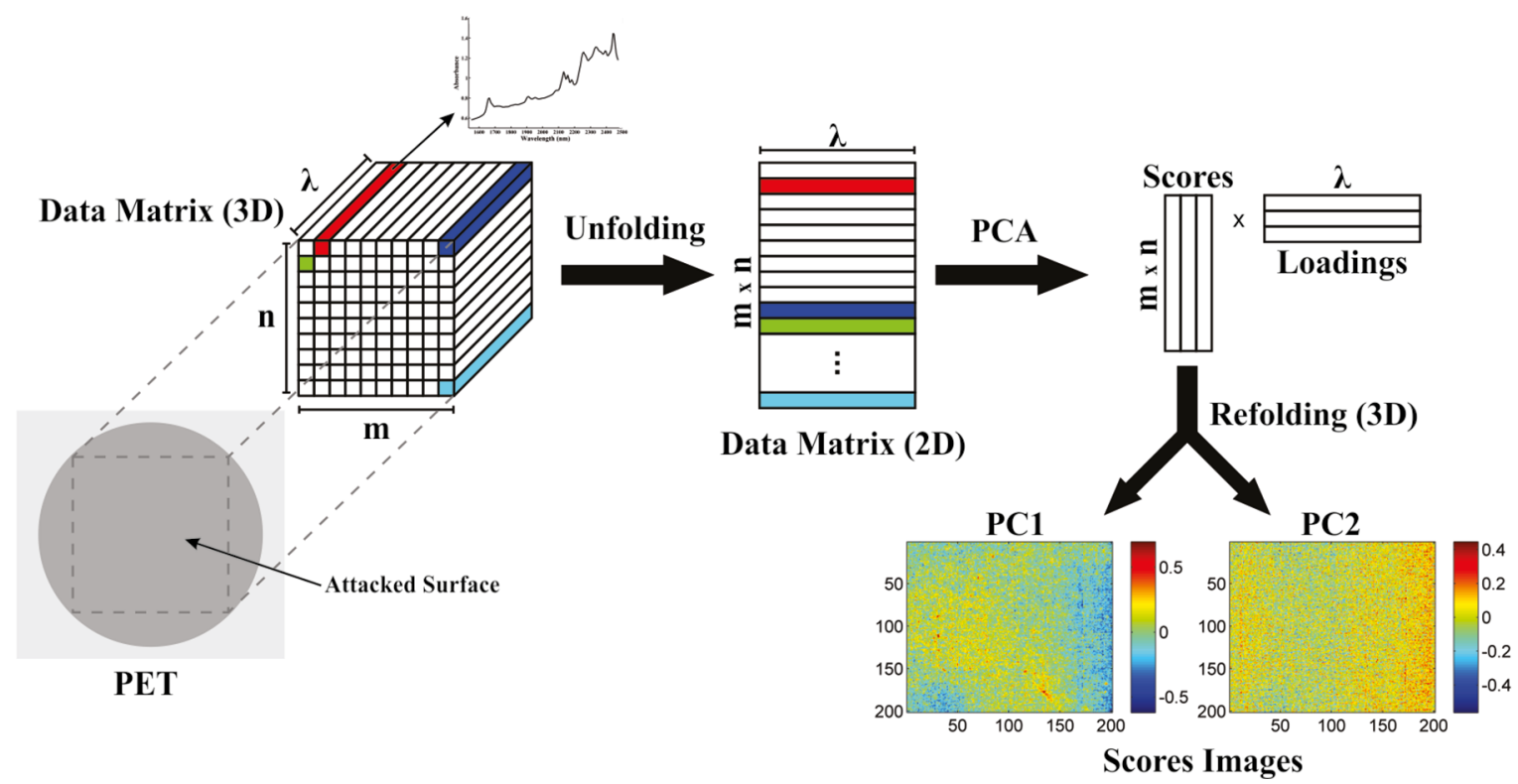

Figure 2. Schematic representation of the PCA in NIR hyperspectral image of the PET showing the hypercube (3D) unfolded into a 2D matrix and the relationship between scores and loadings of the PCA images. 


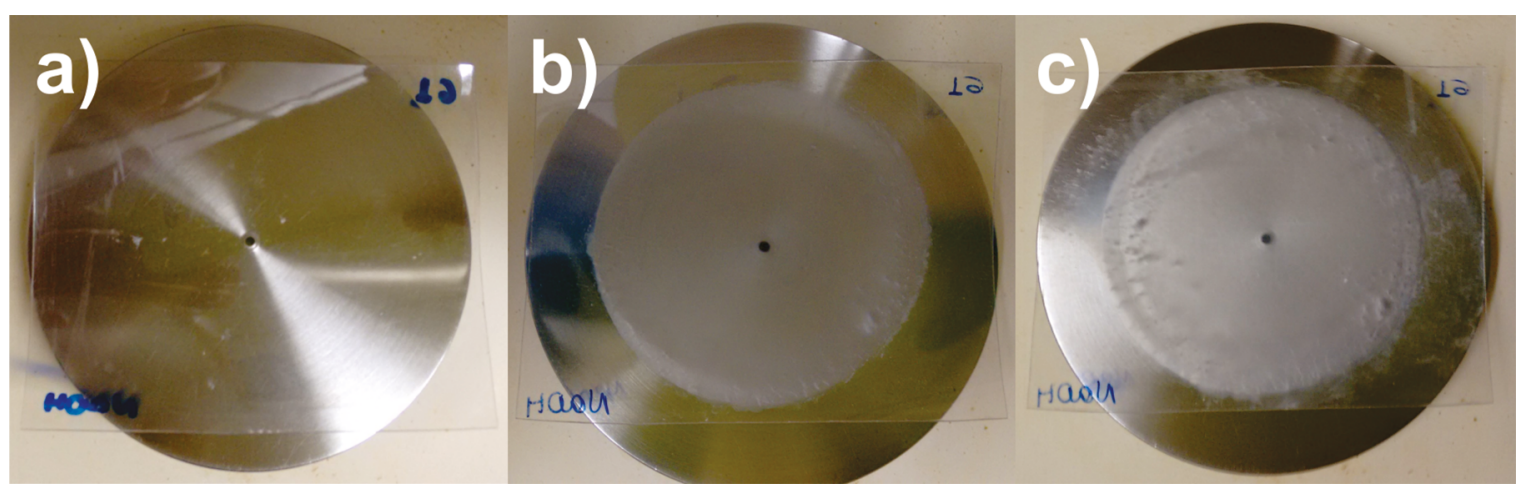

Figure 3. PET samples exposure to $\mathrm{NaOH}$ at (a) time zero, (b) 30 days and (c) 60 days. The metal support was used to better visualize PET changes.

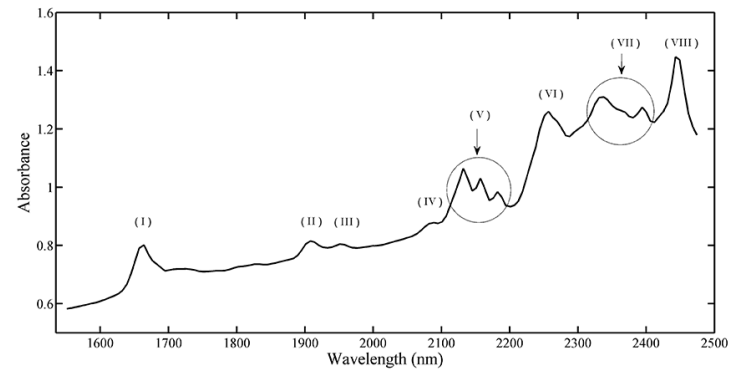

Figure 4. Mean NIR spectrum of PET samples before exposure to the chemical agents.

range from 1.551 to $2.518 \mathrm{~nm}$ was used for further analysis. As can be observed, the first overtone of the $\mathrm{C}-\mathrm{H}$ in aromatics molecules is present around $1.662 \mathrm{~nm}$ (I) and overlapping the first overtone of methylene group stretching. Band (II) can be attributed to the first overtone of the O-H stretching and it can be observed around $1.908 \mathrm{~nm}$. Bands (III) and (IV) are related to the second overtone of the $\mathrm{C}=\mathrm{O}$ stretch and the third overtone of the $\mathrm{C}-\mathrm{O}$ stretch, respectively. Bands $\mathrm{V}$ and VI can be attributed to combination bands of aromatic and combination band of the methylene group, respectively. Finally, overlapping involving methylene groups, aromatic $\mathrm{C}-\mathrm{H}$ and $\mathrm{COO}$ is assigned in bands (VII) and (VIII) ${ }^{34,35}$.

\subsection{Data pre-processing and exploratory analysis}

Among the pre-processing strategies used, SNV with Savitzky-Golay smoothing filtering $\left(2^{\text {nd }}\right.$ order polynomial and 5 window points) evidenced the best visualization of the differences before and after the chemical attack. All the PET samples, exposed during different interval times, were evaluated always in relation to the PET sample before first chemical exposure (time zero). The data sets before and after chemical attack were analyzed using PCA using different spectral ranges (SR1: 1.551 to $2.474 \mathrm{~nm}$; SR2: 1.551 to 2.400 $\mathrm{nm}$ and SR3: 1.801 to $2.251 \mathrm{~nm}$ ). Initially, the images of the samples exposed to the $\mathrm{NaOH}$ solution were evaluated using the spectral range 1 (SR1). Data sets containing the unfolded images of the sample before exposure and after different exposure times were submitted to PCA. The score images of the five first components did not show a clear pattern related to exposure time, neither did they show preferential paths. Then, new PCAs were performed using the SR2, as shown in Figure 5.

As can be observed in the loadings plot, $\mathrm{PC} 1$ (first column in Figure 5) shows variations in the score plots associated with illumination and light scattering. The loadings from PC2 to PC4 are associated with spectral variation of the band centered at $2.332 \mathrm{~nm}$, which is related to aromatics $\mathrm{C}-\mathrm{H}$ stretching in PET. The first four components do not show patterns related to chemical agent exposure. Only in the score image of the fifth component it is possible to observe information associated with the chemical exposure after $24 \mathrm{~h}$ and $96 \mathrm{~h}$ (yellow pixels in the score image). The loadings of PC5 are mainly related to the combination region of (- C - O $+\mathrm{OH})$ band around $1.900 \mathrm{~nm}$. The sample after chemical attack showed higher absorbance values for this band, which can be attributed to ethylene glycol formation, since it is a PET basic hydrolysis product. No preferential paths were observed in these analyses. Therefore, the chemical attack was uniform for the entire surface exposed. For the other chemical exposure times, the same behavior was observed.

PET images after chemical exposure with ethanol and phosphoric acid, ascorbic acid, sodium bicarbonate and citric acid solutions were also evaluated using PCA. As observed with the $\mathrm{NaOH}$ solution, the images did not show clear behavior related to the exposure time employing the SR1. Using the SR2, the loadings of PC2 and PC5 for the samples after exposure with phosphoric and citric acid showed strong influence from the band associated with the same $(-\mathrm{C}-\mathrm{O}+\mathrm{OH})$ combination band around $1.900 \mathrm{~nm}$ (higher absorbance values). For the other chemical agents (ascorbic acid, sodium bicarbonate and ethanol), PCA performed in the SR2 did not show a clear pattern related to exposure time. When the spectral range was reduced to $1.801-2.251 \mathrm{~nm}$ (SR3), differences in the score images related to chemical exposure could be observed, except when ethanol was employed. These variations were observed in the PC2. 
Evaluating the loadings in $\mathrm{PC}$, higher values were also observed in the $(-\mathrm{C}-\mathrm{O}+\mathrm{OH})$ combination region around $1.900 \mathrm{~nm}$. No preferential paths were observed in any of the images analyzed after chemical exposure, indicating that the surfaces were attacked uniformly.

As mentioned, the images of the samples exposed to ethanol did not follow the same pattern as the other chemical agents at different times, as can be seen in the PCA performed with samples after $96 \mathrm{~h}$ exposure to ascorbic acid, citric acid, phosphoric acid, sodium bicarbonate, ethanol and sodium hydroxide (Figure 6). The PC2 score images show clearly that the PET sample exposure to ethanol differed from the others. Evaluation of the PC2 loadings showed that sample exposure to ethanol has lower values, associated with the band at $1.900 \mathrm{~nm}$, indicating no evidence of chemical attack when ethanol was used. Excluding the sample exposed to ethanol, a new PCA was performed. No differences were observed between the samples in the scores and loadings, showing that the chemical attack on the PET was similar under all of these conditions.

Several PCA analyses were performed using samples exposed to the chemical agents for different periods of time to evaluate at what point spectral differences in the surface become evident. PET samples exposed to sodium hydroxide, ascorbic acid, phosphoric acid and citric acid solutions already showed variation in the band at $1.900 \mathrm{~nm}$ after $2 \mathrm{~h}$, demonstrating evidence of chemical attack by these agents. However, the sample exposed to sodium bicarbonate solution only showed spectral variations related to the band at $1.900 \mathrm{~nm}$ after $48 \mathrm{~h}$.

The PCA carried out in the images acquired from the 75 x $55 \mathrm{~mm}$ samples after $96 \mathrm{~h}$ hours of exposure is shown
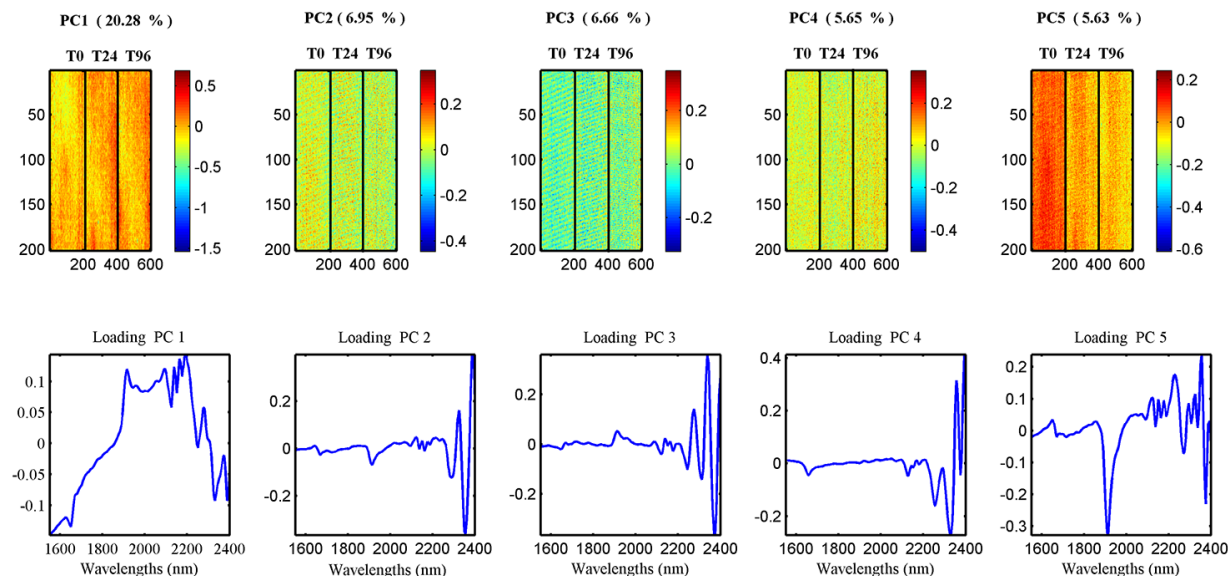

Figure 5. Scores images and loadings of the five first PCs of PET sample after exposure (T0, T24 and T96) to NaOH solution (spectral range: SR2). T0, T24 and T96 correspond to exposure times ( 0 hours, 24 hours and 96 hours), respectively. The axes number refers the spatial dimension (pixels) of each image (200 x 200 pixels) for each time exposure.
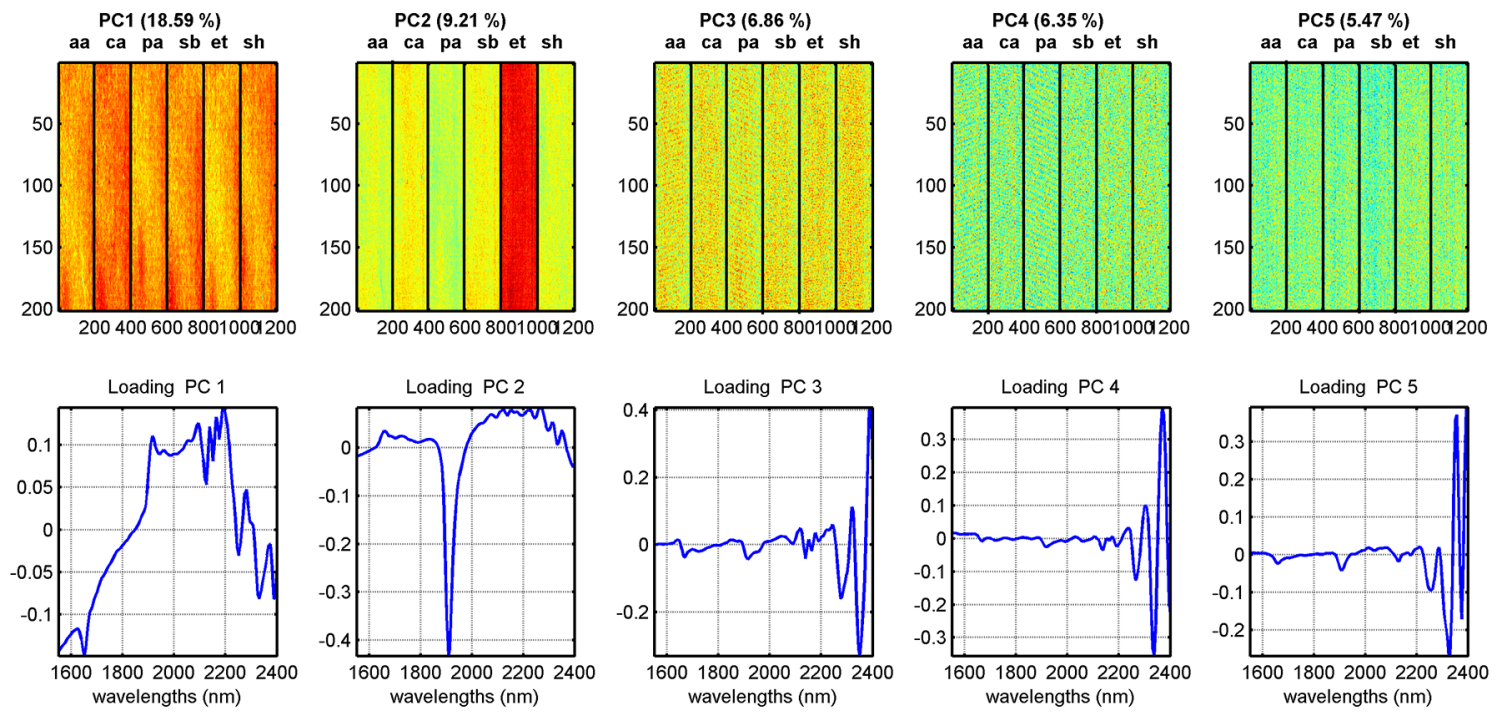

Figure 6. Scores images and loadings of PCA performed with PET samples exposed for $96 \mathrm{~h}$ to chemical agents (order in the illustration: ascorbic acid - aa, citric acid - ca, phosphoric acid - pa, sodium bicarbonate - sb, ethanol - et, and sodium hydroxide - sh). 
in Figure 7. Visual inspection did not identify the areas of the sample exposed to the chemical agents; the score images clearly identified this area (darker blue pixels). The same behavior was observed in the sample after exposure to the chemical agent for shorter periods of time ( $24 \mathrm{~h}$ and $48 \mathrm{~h}$ ).

As the score images do not show preferential paths for the chemical attack (i.e, PET surfaces were uniformly attacked), in order to visualize better the behavior of a sample exposure to different periods of time, a new PCA was performed using the mean spectrum of eight image pixels (selected from the area that has been exposed to the chemical agent) collected randomly from each sample. For this, each data matrix ( $200 \times 200 \times 137)$ was unfolded, obtaining more than 40 mil points per sample. Then, the dots (pixels) were divided into 8 parts and afterwards eight averages were obtained for each sample. The spectral range used in this new PCA was from 1.800 to $2.000 \mathrm{~nm}$ which only depolymerization chemical information was present. Figure 8 shows the score and loading plots of the PCA for the attack with $\mathrm{NaOH}$ solution. The total of variance explained by PC1 and PC2 was $98 \%$. The PCA score plot showed a trend of sample separation according to exposure time. Samples identified as time " 0 " (light blue) were not exposed to the chemical agents and are located in the most negative part PC1 and PC2. Another cluster (green) containing samples exposed during short periods of time (from 2 to $8 \mathrm{~h}$ ) can be observed. Samples exposed to the chemical agents during longer periods (from 24 to 96 hours) tended to form another cluster (red). In the PC1 and PC2 loadings, as expected, the band around $1.900 \mathrm{~nm}$ (with positive values) contributed significantly to differentiation of the samples (Figure 8b). As the loadings related to this band were positive, the samples exposed over longer periods of time had higher absorbance values. The HCA (Figure 8c) evidenced the same clustering pattern, as well as an outlier (one sample exposed for $72 \mathrm{~h}$ ), identified in magenta.

It is important to notice that the radiation in the NIR range can penetrate the PET samples completely to a depth
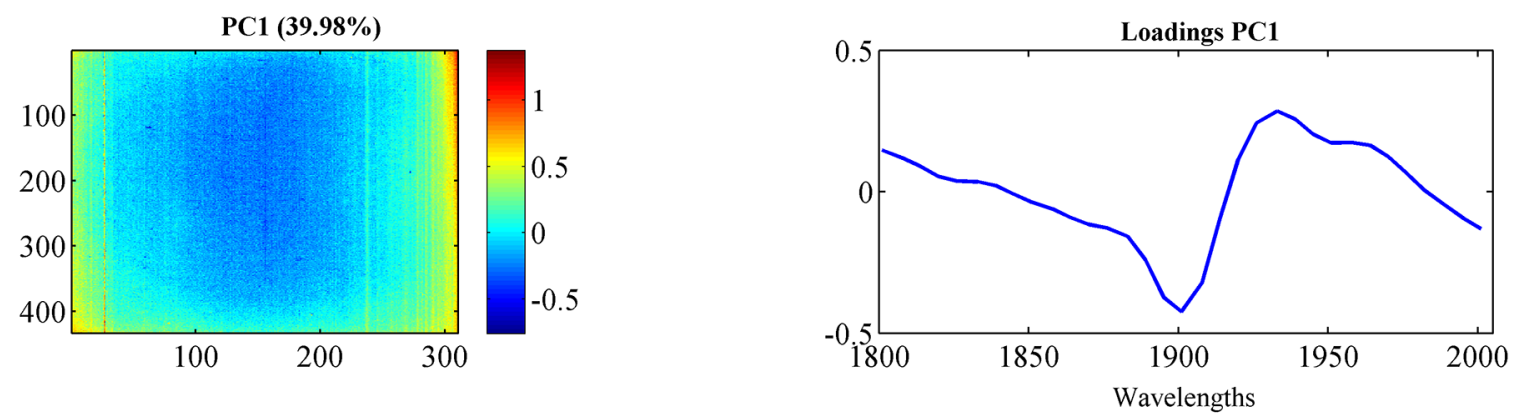

Figure 7. Scores images and loadings of PCA performed on the entire $(75 \times 55 \mathrm{~mm})$ sample after exposure to $\mathrm{NaOH}$ during $96 \mathrm{~h}$. The darker blue pixels show the area exposed.
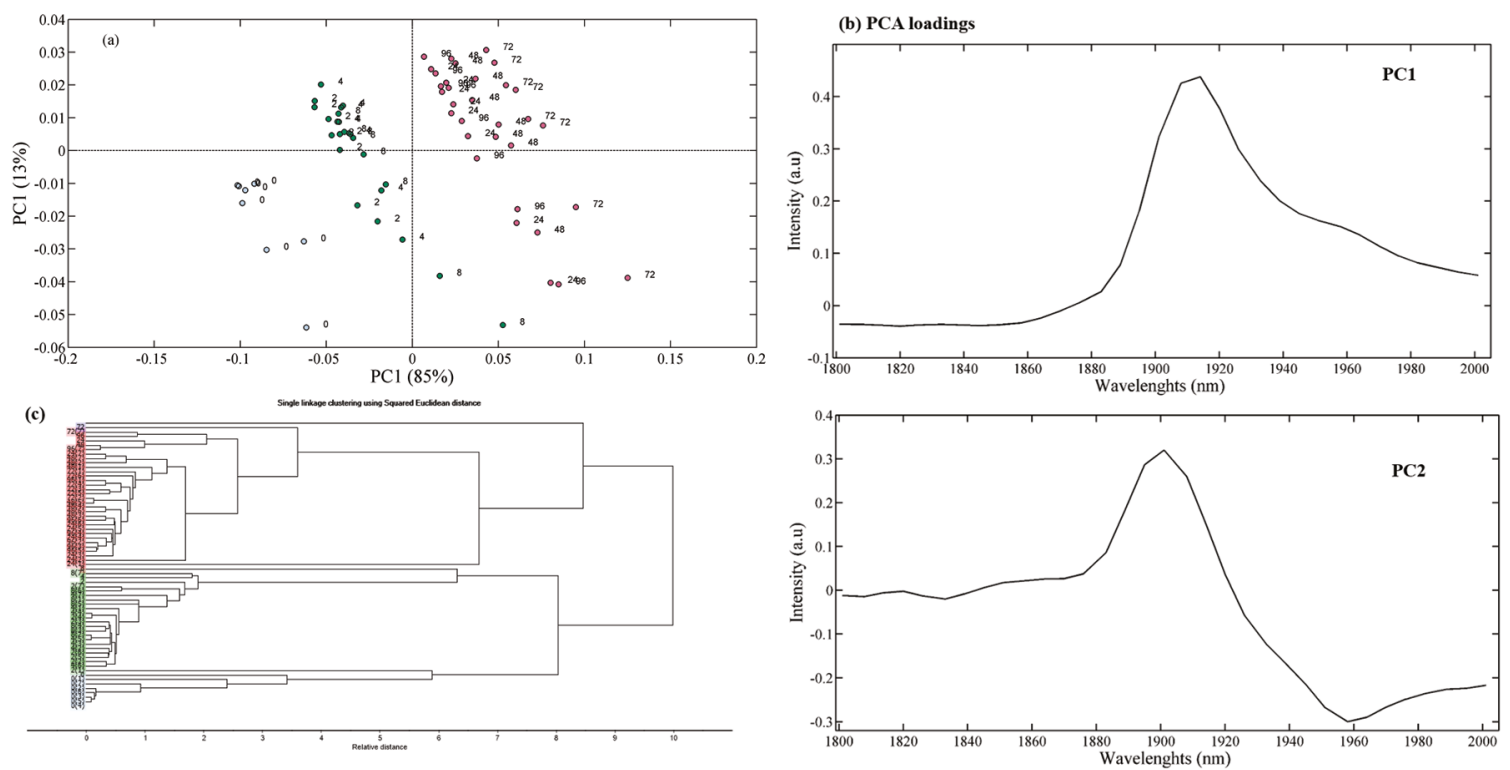

Figure 8. Exploratory analysis performed in the NIR spectra of PET samples exposed to $\mathrm{NaOH}$ during exposure time (a) Scores of PC1 versus PC2, (b) loadings for PC1 and PC2, (c) dendrogram of the HCA. 
around of $800 \mu \mathrm{m}^{36}$. As the thickness of the samples employed in this work was $270 \mu \mathrm{m}$, the spectra showed composition information not only from the surface, but also from the entire region of penetration depth. To confirm this information, a simple experiment was performed. A polypropylene (PP) film was placed below the PET sample and a NIR image was acquired on the top of the PET sample. The resulting mean spectrum (not shown) presented characteristic PP bands, proving that the radiation had penetrated in the PET film.

Attenuated total reflectance spectroscopy in the MIR spectral range was also used to evaluate the spectral variation over the chemical exposure time. Different from the NIR radiation which penetrates completely into the sample thickness, using ATR, the MIR radiation penetrates only to a depth of about 2 micrometers and the spectral information was recorded only for this most superficial layer. The $\mathrm{NaOH}$ solution was selected for this experiment. Figure 9 shows the mean MIR raw spectra (a) and the SNV pre-processed spectra during the chemical exposure time (b).

Figure 9a shows the following absorption bands: at 2.970 $\mathrm{cm}^{-1}(\mathrm{I})$ is attributed to aromatic $\mathrm{C}-\mathrm{H}$ stretching; band at 2.910 $\mathrm{cm}^{-1}$ (II) is associated to aliphatic $\mathrm{C}-\mathrm{H}$ stretching. The group $\mathrm{C}=\mathrm{O}$ stretching is present at $1.714 \mathrm{~cm}^{-1}$ (III). Band (IV) can be attributed to aromatic skeletal stretching. At 1.240 $\mathrm{cm}^{-1}(\mathrm{~V})$ is observed the stretching vibration of $\mathrm{C}(=\mathrm{O})-\mathrm{O}$, characteristic of the ester groups. Bands at $1.096 \mathrm{~cm}^{-1}$ and $1.018 \mathrm{~cm}^{-1}$ (VI) are indicative of an aromatic substitution pattern. At $870 \mathrm{~cm}^{-1}$ (VII) is observed the band of the $\mathrm{C}-\mathrm{H}$ deformation of two adjacent coupled hydrogens on an aromatic ring and at $722 \mathrm{~cm}^{-1}$ (VIII) the band is associated with outof-plane deformation of the two carbonyl substituents on the aromatic ring ${ }^{37,38}$.

As can be seen in Figure 9b, there is a variation in band intensity, even after pre-processing. PCA was performed on the pre-processed dataset. The first two PCs explain $90 \%$ and $6 \%$ of the dataset variability, respectively. Figures $10 \mathrm{a}$ and $10 \mathrm{~b}$ show PET scores and loadings before and after chemical exposure. The PCA score plot showed some tendency toward sample separation over time, but this trend was clear only for a much higher exposure time (after 15 days). The same clustering pattern can be observed in the dendogram shown in Figure $10 \mathrm{c}$. The PCA performed using the NIR spectra region (Figure $8 \mathrm{a}$ ) shows a clearer separation with exposure time, probably due to the higher penetration depth of the NIR radiation in the PET sample.

As depicted in the score plot (Figure 10a), spectra obtained from samples after longer chemical exposition have more negative values in PC1. The loadings of PC1 (Figure 10b) showed high positive values associated to the bands at 1.714 and $1.244 \mathrm{~cm}^{-1}$, which can be attributed to the carbonyl and $\mathrm{C}$ (O) - O stretches. Then, with longer exposure time there was
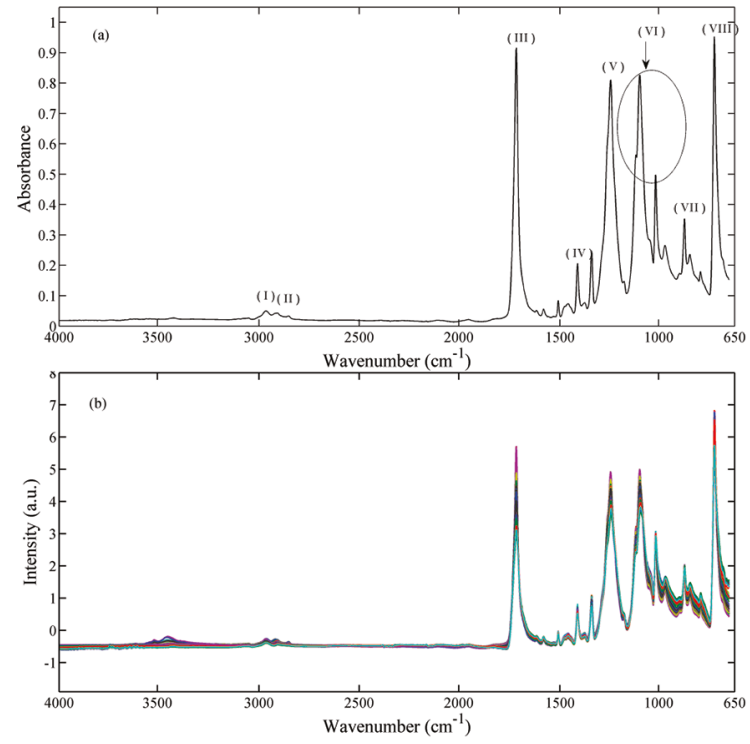

Figure 9. (a) Mean MIR raw spectra before chemical attack and (b) after exposure to $\mathrm{NaOH}$ (pre-processed with SNV).

a decrease in the absorbance values of these bands. Holland and Ray (2002) also reported a decrease of the carbonyl band in the PET spectra after degradation ${ }^{38}$. This decrease probably can be associated with partial depolymerization of the PET in contact with $\mathrm{NaOH}$ solution. PC2 scores plot evidenced that samples after 60 days of chemical exposure had higher negative values. The loadings of PC2 (Figure 10b) showed that this component was influenced mainly by the band at $722 \mathrm{~cm}^{-1}$ associated with the out of plane deformation of the two carbonyl substituents on the aromatic ring. This also can be attributed to the partial depolymerization of the PET sample with $\mathrm{NaOH}$, which occurred directly in the carbonyl group bonded to the aromatic ring, transforming the ester into sodium terephthalate. That transformation can also be confirmed by the whitish color observed in samples treated with $\mathrm{NaOH}$, as reported in the visual PET analysis.

As mentioned, destructive techniques such as scanning electron microscopy (SEM), Differential Scanning Calorimetry (DSC) and Thermogravimetric Analysis (TGA) have been used to evaluate effects of a chemical attack in polymeric materials. The samples exposed to $\mathrm{NaOH}$ were also analyzed using these techniques. The DSC and TGA were not efficient as their results did not show any significant difference either before or after exposure to $\mathrm{NaOH}$. The same behavior has been reported by Rosmaninho et $a l^{8}$. The PET sample before and after 30 days of exposure to $\mathrm{NaOH}$ was also analyzed by scanning electron microcopy (SEM) and the results can be seen in Figure 11. Before exposure (Figure 11a), the sample shows a very regular surface. After exposure (Figure 11b), the surface shows a more porous and rougher aspect, as a result of the depolymerization. 

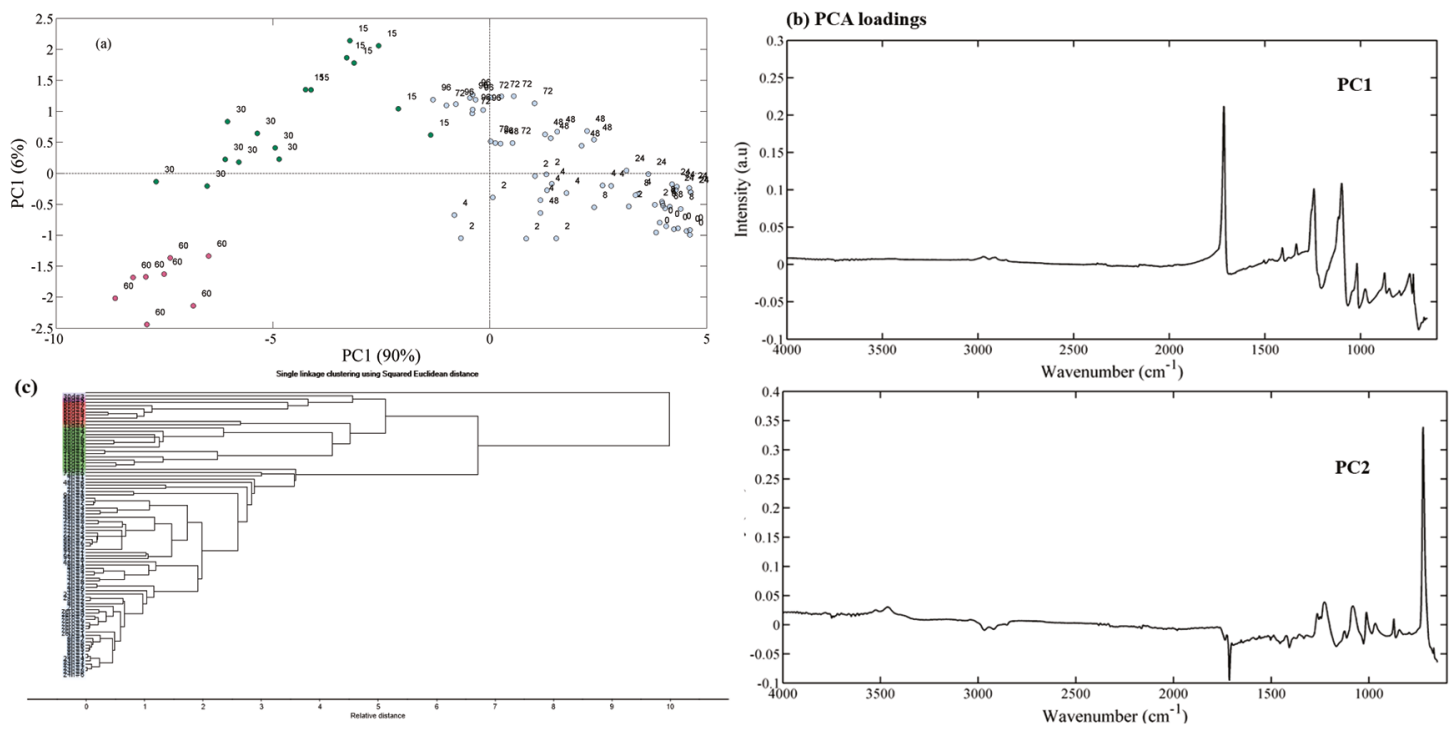

Figure 10. Exploratory analysis performed in the PET MIR spectra before and after chemical attack with $\mathrm{NaOH}$ at different exposure times (a) PC1 versus and PC2 score plot, (b) PC1 and PC2 loadings and (c) the dendogram of the HCA.

\section{Conclusions}

Changes in bottle PET surface after chemical attack by different chemical agents at room temperature were successfully investigated using infrared spectroscopy and, in particular, near infrared hyperspectral imaging. The accessory employed ensures that the chemical agent was in contact only with the surface of the samples (and not with the lateral areas). Preferential paths where the chemical agents penetrated the PET film were not observed by NIR hyperspectral images. Evaluation of the score plots of the PCA performed with the HSI-NIR made it possible to identify the depolymerization of PET through the combination band $(-\mathrm{C}-\mathrm{O}+\mathrm{OH})$, characterizing the formation of the ethylene glycol. PET samples exposed to chemical agents (sodium hydroxide, ascorbic acid, phosphoric acid and citric acid solutions) showed evidence of chemical attack after $2 \mathrm{~h}$. Areas of the samples exposed to the chemical agents could be clearly identified from the NIR images constructed with PCA scores. Variations in the intensity of the bands of ATR spectra, which provided information only about the superficial layer of the polymeric material (around $2 \mu \mathrm{m}$ ), showed evidence of chemical attack by $\mathrm{NaOH}$ and superficial depolymerization of the PET after only 15 days of exposure. The higher penetration depth of the NIR radiation associated with the spatial information provided by the image system showed advantages over the MIR-ATR spectroscopy. The methodology proposed enables observation of the chemical changes on the polymer after chemical agent exposure at room temperature, as well as the absence of preferential paths, in a very fast and nondestructive way. This can be useful for other case studies of polymer attack/degradation and/or to reveal areas of degradation not identified visually.

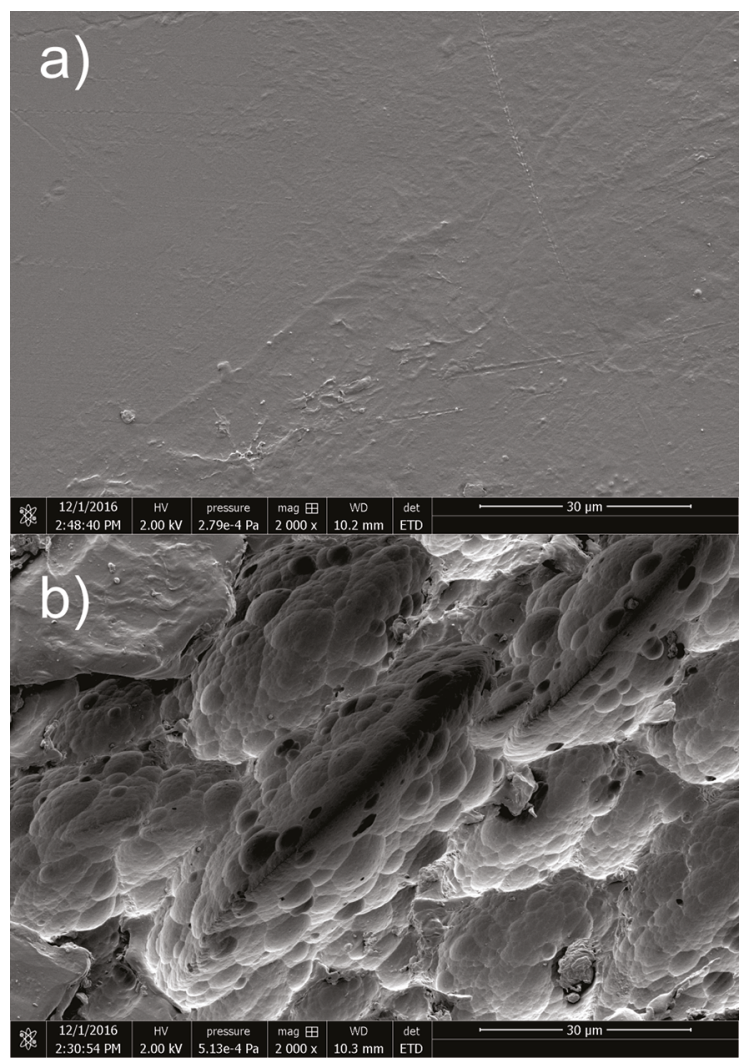

Figure 11. SEM of the PET sample (a) before and (b) after chemical attack with $\mathrm{NaOH}$ for 30 days.

\section{Acknowledgements}

This work was supported by the INCTAA (Processes $n^{\circ}$.: CNPq 573894/2008-6; FAPESP 2008/57808-1), NUQAAPE -FACEPE (APQ-0346-1.06/14), CNPq, FACEPE, CAPES. 
We also thank to GIA/IQ/UNICAMP and Empraba - Centro Nacional de Pesquisa de Algodão for allowing us to use the infrastructure of laboratory, Viviane Fonseca Caetano for the preliminary experiments and Eduardo maia Paiva for producing Figures 1 and 2. The English text of this paper has been revised by Sidney Pratt, Canadian, MAT (The Johns Hopkins University), RSAdip - TESL (Cambridge University).

\section{References}

1. Teófilo ET, Melo RN, Silva SML, Rabello MS. Stress cracking e ataque químico do PET em diferentes agentes químicos. Polímeros. 2009;19(3):202-211.

2. Wypych G. Environmental Stress Cracking. In: Wypych G. Handbook of Material Weathering. Toronto: ChemTec Publishing; 2013. p. 731-753.

3. Moskala EJ. A fracture mechanics approach to environmental stress cracking in poly(ethyleneterephthalate). Polymer. 1998;39(3):675-680.

4. Al-Saidi LF, Mortensen K, Almdal K. Environmental stress cracking resistance. Behaviour of polycarbonate in different chemicals by determination of the time-dependence of stress at constant strains. Polymer Degradation and Stability. 2003;82(3):451-461.

5. Morrison ED, Malvey MW, Johnson RD, Hutchison JS. Mechanism of stress cracking of poly(ethylene terephthalate) beverage bottles: A method for the prevention of stress cracking based on water hardness. Polymer Degradation and Stability. 2010;95(4):656-665.

6. Mancini SD, Zanin M. Influência de Meios Reacionais na Hidrólise de PET Pós-Consumo. Polímeros. 2002;12(1):34-40.

7. Genta M, Goto M, Sasaki M. Heterogeneous continuous kinetics modeling of PET depolymerization in supercritical methanol. The Journal of Supercritical Fluids. 2010;52(3):266-275.

8. Rosmaninho MG, Jardim E, Moura FCC, Ferreira GL, Thom V, Yoshida MI, et al. Surface hydrolysis of postconsumer polyethylene terephthalate to produce adsorbents for cationic contaminants. Journal of Applied Polymer Science. 2006;102(6):5284-5291.

9. Muller D, Israelsohn-Azulay O. Chemical Contamination of Soft Drinks in Sealed Plastic Bottles by Environmental Stress Cracking. Journal of Forensic Sciences. 2009;54(1):128-131.

10. Espinoza García K, Navarro R, Ramírez-Hernández A, Marcos-Fernández Á. New routes to difunctional macroglycols using ethylene carbonate: Reaction with bis-(2-hydroxyethyl) terephthalate and degradation of poly(ethylene terephthalate). Polymer Degradation and Stability. 2017;144:195-206.

11. Fonseca TG, de Almeida YMB, Vinhas GM. Chemical Recycling of Post-consumer PET: Structural Characterization of terephthalic acid and the effect of Alkaline Hydrolysis at low temperature. Polímeros. 2014;24(5):567-571.

12. Ng R, Zhang X, Liu N, Yang ST. Modifications of nonwoven polyethylene terephthalate fibrous matrices via $\mathrm{NaOH}$ hydrolysis: Effects on pore size, fiber diameter, cell seeding and proliferation. Process Biochemistry. 2009;44(9):992-998.
13. Liu Y, He T, Gao C. Surface modification of poly(ethylene terephthalate) via hydrolysis and layer-by-layer assembly of chitosan and chondroitin sulfate to construct cytocompatible layer for human endothelial cells. Colloids and Surfaces B: Biointerfaces. 2005;46(2):117-126.

14. Çinar ME, Kar F. Characterization of composite produced from waste PET and marble dust. Construction and Building Materials. 2018;163:734-741.

15. Pandey JK, Raghunatha Reddy K, Pratheep Kumar A, Singh RP. An overview on the degradability of polymer nanocomposites. Polymer Degradation and Stability. 2005;88(2):234-250.

16. Mukherjee S, Gowen A. A review of recent trends in polymer characterization using non-destructive vibrational spectroscopic modalities and chemical imaging. Analytica Chimica Acta. 2015;895:12-34.

17. Ozzetti RA, De Oliveira Filho AP, Schuchardt U, Mandelli D. Determination of tacticity in polypropylene by FTIR with multivariate calibration. Journal of Applied Polymer Science. 2002;85(4):734-745.

18. Ghita OR, Baker DC, Evans KE. An in-line near-infrared process control tool for monitoring the effects of speed, temperature, and polymer colour in injection moulding. Polymer Testing. 2008;27(4):459-469.

19. Caetano VF, Vinhas GM, Pimentel MF, Simões SDS, de Araújo MCU. Prediction of mechanical properties of poly(ethylene terephthalate) using infrared spectroscopy and multivariate calibration. Journal of Applied Polymer Science. 2013;127(5):34413446.

20. Kassouf A, Ruellan A, Jouan-Rimbaud Bouveresse D, Rutledge DN, Domenek S, Maalouly J, et al. Attenuated total reflectancemid infrared spectroscopy (ATR-MIR) coupled with independent components analysis (ICA): A fast method to determine plasticizers in polylactide (PLA). Talanta. 2016;147:569-580. DOI:http://dx.doi.org/10.1016/j.talanta.2015.10.021

21. Prats-Montalbán JM, de Juan A, Ferrer A. Multivariate image analysis: A review with applications. Chemometrics and Intelligent Laboratory Systems. 2011;107(1):1-23. DOI:http:// dx.doi.org/10.1016/j.chemolab.2011.03.002

22. Amigo JM, Babamoradi H, Elcoroaristizabal S. Hyperspectral image analysis. A tutorial. Analytica Chimica Acta. 2015;896:3451.

23. Gosselin R, Rodrigue D, Duchesne C. On-line prediction of crystallinity spatial distribution across polymer films using NIR spectral imaging and chemometrics methods. The Canadian Journal of Chemical Engineering. 2008;86(5):869-878.

24. Serranti S, Gargiulo A, Bonifazi G. The Utilization of Hyperspectral Imaging for Impurities Detection in Secondary Plastics. The Open Waste Management Journal. 2010;3:5670.

25. Unger M, Sedlmair J, Siesler HW, Hirschmugl C. 3D FTIR imaging spectroscopy of phase-separation in a poly(3hydroxybutyrate)/poly(1-Lactic acid) blend. Vibrational Spectroscopy. 2014;75:169-172. DOI: http://dx.doi. org/10.1016/j.vibspec.2014.07.007 
26. Moroni M, Mei A, Leonardi A, Lupo E, La Marca F. PET and PVC Separation with Hyperspectral Imagery. Sensors (Basel). 2015;15(1):2205-2227.

27. Caetano VF, Rodrigues e Brito L, Rohwedder JJR, Pasquini C, Pimentel MF, Vinhas GM. Determination of diethyleneglycol content and number of carboxylic end groups in poly(ethylene terephthalate) fibers using imaging and conventional near infrared spectroscopy. Polymer Testing. 2016;49:15-21.

28. Muroga S, Hikima Y, Ohshima M. Visualization of hydrolysis in polylactide using near-infrared hyperspectral imaging and chemometrics. Journal of Applied Polymer Science. 2018;135(8):45898.

29. Morrison ED, Malvey MW, Johnson RD, Anacker JL, Brown KA. Effect of chemical environments on stress cracking of poly(ethylene terephthalate) beverage bottles. Polymer Testing. 2008;27(6):660-666.

30. Berlinet C, Brat P, Ducruet V. Quality of orange juice in barrier packaging material. Packaging Technology and Science. 2008;21(5):279-286.

31. Bacigalupi C, Maurey A, Boutroy N, Peyron S, Avallone S, Chalier P. Changes in nutritional value of a multi-vitamins fortified juice packed in glass and standard PET bottles. Food Control. 2016;60:256-262.
32. Penczek S, Pretula J, Kubisa P, Kaluzynski K, Szymanski R. Reactions of H3PO4 forming polymers. Apparently simple reactions leading to sophisticated structures and applications. Progress in Polymer Science. 2015;45:44-70.

33. Beebe KR, Pell RJ, Seasholtz MB. Chemometrics: A Practical Guide. New York: John Wiley \& Sons; 1998.

34. Miller CE, Eichinger BE. Determination of Crystallinity and Morphology of Fibrous and Bulk Poly(ethylene terephthalate) by Near-Infrared Diffuse Reflectance Spectroscopy. Applied Spectroscopy. 1990;44(3):496-504.

35. Workman J Jr, Weyer L. Practical Guide and Spectral Atlas for Interpretive Near Infared Spectroscopy. 2nd ed. Boca Raton: CRC Press; 2012.

36. Haanstra WG, Hansen W, Huys MJG, Kip BJ, Palmen P, Roumen $\mathrm{J}$, et al. Information Depth of Vis-NIR Light in Polyethylene Films Using Transmission and Reflectance Measurements. Applied Spectroscopy. 1998;52(6):863-868.

37. Golshaei P, Güven O. Chemical modification of PET surface and subsequent graftcopolymerization with poly(N-isopropylacrylamide). Reactive and Functional Polymers. 2017;118:26-34.

38. Holland BJ, Hay JN. The thermal degradation of PET and analogous polyesters by thermal analysis-Fourier transform infrared spectroscopy. Polymer. 2002;43(6):1835-1847. 